Voix et Images

voixetimages

\title{
L’autorité du sentiment dans Les Fiancés de 1812
}

\section{André Sénécal}

Volume 7, numéro 1, automne 1981

\section{Adrien Thério}

URI : https://id.erudit.org/iderudit/200310ar

DOI : https://doi.org/10.7202/200310ar

Aller au sommaire du numéro

Éditeur(s)

Les Presses de l'Université du Québec

ISSN

0318-9201 (imprimé)

1705-933X (numérique)

Découvrir la revue

Citer cet article

Sénécal, A. (1981). L’autorité du sentiment dans Les Fiancés de 1812. Voix et Images, 7(1), 169-175. https://doi.org/10.7202/200310ar d'utilisation que vous pouvez consulter en ligne.

https://apropos.erudit.org/fr/usagers/politique-dutilisation/ 


\title{
L'autorité du sentiment dans Les Fiancés de 1812
}

\author{
par André Sénécal
}

Les quelques critiques qui se sont penchés sur le roman de Joseph Doutre l'ont vite relégué au rang des narrations invraisemblables. Même s'ils se sont montrés plus fidèles au texte qu'un Séraphin Marion ${ }^{1}$, les commentateurs les plus récents, David Hayne par exemple ${ }^{2}$, se sont contentés de relever l'ahurissant imbroglio du récit sans chercher à en découvrir la portée didactique. En accord avec la critique, on doit avouer que la narration événementielle des Fiancés est le produit d'une imagination fiévreuse. Le hasard remonte constamment les ressorts de l'action et les enlèvements, les dissimulations, retardent indûment un dénouement d'ailleurs artificiel. Le millionnaire SaintFelmar s'oppose au mariage de sa fille avec Gonzalve de R... parce qu'il est jaloux des titres de noblesse de la famille du jeune homme. Profitant de l'absence du héros, le parvenu ordonne à Louise d'épouser un vil prétendant. Déguisée en jeune homme, l'héroïne fuit la maison paternelle et se dirige vers le camp de la milice canadienne à Chateauguay pour y rejoindre son amant. Un cruel hasard sépare le couple. Louise est capturée par un brigand et, après maintes péripéties, on la retrouve derrière les lignes de l'ennemi américain où Saint-Felmar la rejoindra pour la persuader de le suivre. Le père a tôt fait de persécuter les amants et, de nouveau, il pousse sa fille dans les bras d'un être immonde. Cette fois, une longue maladie la sauvera d'un mariage forcé. Finalement, Gonzalve dupe le père monstrueux et s'enfuit avec Louise. Ce n'est que neuf ans plus tard, après avoir appris que son gendre lui avait autrefois sauvé la vie, que Saint-Felmar se réconcilie avec le couple.

Dans la deuxième partie du roman, Doutre insère les mémoires de Gustave, le frère de Louise. Ce récit d'aventures extraordinaires n'a qu'un rapport ténu avec l'intrigue sentimentale, mais il est rempli d'exploits pittoresques. Ce frère qu'on croyait mort revient juste à temps pour aider Louise à détromper leur père. Ce n'est pas la première fois que Gustave participe à l'intrigue. C'était lui, Le Grand, chef des brigands, qui avait enlevé sa propre sœur! Les mémoires «Six années de ma vie», nous racontent sa carrière d'aventurier. Proscrit, victime du sort, Gustave traîne son mal du siècle à travers l'Europe. On le retrouve en Grèce où sa bravoure lui gagne la main d'Alpina. 
Après la capture de sa femme par les Turcs, il apprend qu'elle préfère l'esclavage du sérail au suicide honorable. Désabusé, il reprend sa vie de fortune et devient l'éminence grise d'une bande de nobles malfaiteurs. Entraîné par le destin, il devient Le Grand, chef de la douce rapine. Il interdit le meurtre, protège les faibles. C'est au cours d'une visite à ses cohortes canadiennes qu'il capture sa propre sœur et que, la prenant pour un homme, il lui fait des avances sexuelles. Cette rencontre d'une âme innocente l'impressionne fortement et, quelque temps après, ayant retrouvé les traces de sa famille, il donne sa fortune aux pauvres et s'intègre à la bonne société.

Comme on le voit, le jeune romancier de dix-neuf ans intercalle des aventures inouies à des allusions aux exploits historiques de Chateauguay pour nous plonger dans l'admiration, l'effroi et le mystère. Bien que certains détails portent sur la Guerre de 1812 et contribuent à une mise en scène ımpressionnante, Doutre n'a pas écrit un roman historique. D'ailleurs, quoi qu'en disent certains critiques, l'auteur n'avait pas cette intention: "L'historien sera quelquefois choqué du peu de respect que nous avons pour la vérité. ... Notre ignorance nous eût peut-être restreint dans un travail d'une autre nature. Mais nous nous sommes contenté de quelque relation verbale sur les événements historiques avec lesquels nous lions notre nouvelle» (p. $x i x)^{3}$. Doutre exploite une historiographie populaire déjà romanesque. Ses digressions sur l'importance de l'escarmouche de Chateauguay reprennent les panégyriques de Mermet et de Bibaud qui ne seront corrigés que par I'Histoire du Canada de Garneau. Le romancier retient des anecdotes véridiques (l'exécution de deux voltigeurs par exemple), mais les aventures qu'il brode autour de détails vrais sortent tout d'une pièce de son imagination. Les guérillas du colonel de Salaberry et les troupes américaines sont transformés en militaires en dentelle qui observent des galanteries de salon. Les bribes d'histoire se perdent dans un tissu d'exploits invraisemblables.

L'action et la mise en scène des épisodes qui ne se rapportent pas au théâtre de 1812 ne sont pas plus authentiques. On s'étonne de retrouver un château sur une île du lac Saint-Louis, des loges de brigands dans les caves d'un sénateur américain! Alphonse a pour père un baron de Montréall Le vérifiable n'existe pas, la couleur locale non plus. Les rares descriptions du décor sortent toutes figées de la rhétorique néo-classique d'un collégien : «Les rayons du soleil venaient de faire place au crépuscule qui s'annonçait par l"apparition du char illuminé de la lune" (p. 103). Les portraits physiques et moraux sont eux aussi artificiels. La sensiblerie de Gonzalve, l'audace féministe de Louise, l'âme sombre de Gustave, la caricature du père monstrueux, ces traits et tant d'autres sont empruntés aux conventions romanesques prébalzaciennes.

Tout comme les fils de l'intrigue, la leçon didactique des Fiancés de 1812 est tirée de la morale des Baculard d'Arnaud et des Loaisel de Tréogate. Malgré ce manque d'originalité, l'auteur n'accuse pas moins son désir de nous offrir une leçon développée sur le rôle du sentiment dans le bonheur. Les person- 
nages et leurs aventures illustrent une éthique rationaliste basée sur I'hédonisme. Dès sa préface, Doutre se prévaut de l'apport moral du roman en citant l'influence bienfaisante de l'œuvre d'Eugène Sue et en glissant un avertissement à la manière des auteurs libertins: «On rencontrera quelque part des discussions qui n'auront pas beaucoup d'intérêt pour un grand nombre. Mais elles seront brèves, et si les quelques personnes auxquelles nous nous adressons nous comprennent, nos vues seront remplies" (p. $x x$ ). En fait, ces "discussions qui n'auront pas beaucoup d'intérêt pour un grand nombre" tiennent peu de place dans cet ouvrage volumineux. Vers le début de la deuxième partie, Doutre ouvre une parenthèse de quelques pages (pp. 355-364) pour prêcher la tolérance et fustiger l'hypocrisie des dévots. Le romancier stigmatise les tartuffes de son milieu. II se sert des traits les plus noirs pour nous peindre des pharisiens qui affichent une morale orthodoxe, mais qui sont des cloaques de tous les vices: "Que ne peut-on sonder les secrets de ces hommes machiavéliquement hypocrites! Quelles fraudes cachées! Quels crimes ignorés! Quelle immoralité secrète! Plus le méchant se cache, plus ses coups sont terribles" (p. 363). Le futur chef de file de l'Institut Canadien nous offre en contraste une image d'Épinal du libre penseur déiste qu'il identifie à Gonzalve, son héros. Le romancier nous montre deux «bons chrétiens", l'un qui cite Bossuet, l'autre qui invoque Voltaire. Ensemble, ils vont à «l'office divin» pour ensuite passer au théâtre. Nous sommes loin du troupeau de Mgr Bourget. Malgré sa brièveté, cette digression nous dévoile le lieu idéologique du narrateur et nous suggère l'origine rationaliste de ses idées sur le bonheur et la vertu.

L'intrigue principale, celle de l'amour contrarié de Louise et de Gonzalve, proclame l'origine sensuelle du sentiment et sa primauté dans le domaine moral. Gonzalve, que l'auteur nous présente comme un paragon de vertus, affiche franchement son hédonisme: «Depuis le moment où l'âge m'a placé dans la société, je n'ai connu d'autre maître que l'amour. Dans toutes les circonstances où m'a mené depuis le cours de la vie, il a été le moteur de toutes mes actions. Et si je dois juger la généralité des hommes par moi-même, je ne craindrai pas de dire que l'homme est créé pour aimer» (p. 89). Cet amour n'est pas platonique. Gonzalve éprouve "les puissantes passions qu'inspirent les charmes d'un sexe enchanteur» (p. 49). Devant un autre initié, sans fausse honte, le jeune amant n'hésite pas à louer Louise "dans son état le plus simple, dénuée d'ornements et de parure..." (p. 49). "Doué d'une sensibilité extrême," le jeune militaire s'abandonne à des souvenirs voluptueux: "Quand elle me disait ces mots enchanteurs : "Je suis à toi pour la vie," comme je voyais se balancer mollement cette belle poitrine qui réflétait sa couleur sur un cou d'albâtre...» (pp. 50-51). L'appétit charnel joue un rôle aussi prééminent dans la vie de Louise. Sa lettre nous apprend pourquoi elle transgresse les "liens sacrés" entre père et fille. En révolte contre une volonté despotique, elle obéit à un instinct impérieux que la religion et la société sanctionnent. Citant un précepte évangélique, Louise admoneste son père: "Ne vais-je pas sur les traces de l'époux que le ciel et mon cœur m'ont choisi?" (pp. 72-73). 
L'intrigue des Fiancés de 1812 établit non seulement la primauté de l'appétit sexuel, mais encore, son rôle de grâce originelle. Ce sont les attraits de Louise plutôt que des remords qui éveillent Gustave et Brandsome à la vertu. Ce dernier nous est d'abord présenté comme un mondain dépravé, mais, grâce au pouvoir rédempteur de Louise, ses penchants grossiers se transformeront en hédonisme vertueux. Brandsome est foudroyé par la beauté de l'amante de Gonzalve et ce ne sont que les bienséances de l'amitié qui retiennent ses instincts lubriques. Mystérieusement, le sentiment transfigure ces transports en chaste admiration. Méditant sur sa première rencontre avec Louise, l'Américain écrit à Alphonse: «Depuis ce moment je ne vis que de songes et de souvenirs. Mon esprit erre sans cesse sur les premières catastrophes de mon cour jusqu'alors insensible» (p. 299). Séparé de "son ange», l'officier se contentera de «l'adorer respectueusement et comme un objet sacré » (p. 299). Éveillé à la vertu, Brandsome épousera la sœur d'Alphonse pour pouvoir rejoindre le petit cercle d'initiés de Louise et de Gonzalve. La bienfaisance exaltée et l'épicurisme qui caractérisent ce cénacle ne sont pas sans nous rappeler certains crédos euphoriques de la littérature maçonnique du dixhuitième siècle.

L'histoire de Gustave nous apporte une autre illustration du pouvoir du sentiment. C'est encore avec Louise qui, par le truchement des sens, réveille une âme au bonheur. Exemple des plus extrêmes du rapport entre la grâce et les sens puisque Gustave fait des avances à sa propre sœur déguisée en jeune homme. En se servant de l'interdit, Doutre veut souligner que le sentiment, même lorsque son origine sensible est incestueuse ou homosexuelle, a le pouvoir prodigieux de restaurer l'homme à sa condition pristine. Le romancier n'essaie pas de nous expliquer le pouvoir du sentiment; il se contente d'en proclamer l'autorité ${ }^{4}$. La vertu se forge dans l'effusion de l'âme sensible. L'acuité morale dépend, à l'origine d'une prise de conscience, d'une épiphanie. Mû par le sentiment, l'homme se rend compte de son éloignement des autres et des égarements de son cœur. Malgré des années de brigandage et de débauche, Gustave peut jouir d'un bonheur facile et immédiat. Créature de l'énergie, victime du destin, le frère de Louise invoque la fatalité: "J'étais né pour le bien. Le sort m'a détourné de la voie que m'avait tracée la nature. $J$ ai été méchant par vicissitudes et par entraînement du destin " (p. 388). Doutre fait l'apologie d'unẹ âme noble au sein de la dégradation. Le cercle d'initiés qui écoute sa confession ne le condamne pas. Même sa sœur qui l'a connu dans des circonstances des plus équivoques, n'est pas repoussée par son passé troublant. Loin de là; la troupe joyeuse reprend la scène inquiétante de la rencontre entre frère et soeur pour la transformer en jeu de société. Doutre s'enthousiasme pour les illusions de l'optimisme rationaliste. Dans le monde que le jeune romancier imagine, le bonheur et la vertu se concilient sans peine avec les instincts les plus hédonistes de l'homme. L'auteur repousse la dualité chrétienne entre âme et matière, entre distinct et grâce. On ne retrouve pas de concepts d'expiation ou de faute originelle. Le mal, qui n'est jamais inexorable, se réduit à la misanthropie. 
La misanthropie, c'est le trait essentiel du personnage de Saint-Felmar. La haine, nourrie par l'envie, frustre son cceur et le rend insensible. Éloigné de l'amour des autres, le millionnaire devient un père monstrueux. Mais dans le système utopique de Doutre, il n'existe pas de méchants impavides. Inéluctablement, la bienfaisance aura raison de la haine la plus inique. Avec une foi aveugle dans la grâce du sentiment, les amants attendront la conversion de Saint-Felmar pendant plus de neuf ans. Au cours de cette longue épreuve, Gonzalve répondra aux injures et aux noires visées de son beau-père en offrant l'autre joue. Mème après avoir sauvé son persécuteur d'une mort certaine, le jeune colonel refusera de lui révéler son identité. La bienfaisance demande un altruisme total, une confiance aveugle. Dans son magistral L'/dée du bonheur au XVIIIe siècle, Robert Mauzi a bien défini cette exaltation de la bienfaisance et son rôle dans l'éthique rationaliste du siècle des lumières: «Tout indique que l'essence de l'homme est d'être sociable, qu'il ne peut exister séparé des autres. L'art d'être heureux consiste à prendre conscience de cette vérité. La morale, à la considérer du point de vue d'autrui ${ }^{5}$. C'est exactement ce que nous démontre l'intrigue sentimentale des fiancés. La morale est un art de plaire.

Cependant, c'est un art de plaire réservé à des âmes «favorisées de la nature et de la fortune". L'épisode des amours burlesques de Bossendos et de Mathon, domestiques de Saint-Felmar, nous avertit que la pauvreté rend le bonheur philosopique impossible. Êtres grotesques dont le physique difforme reflète l'âme embryonnaire, ils ne peuvent jouir que des plaisirs grossiers de la bête. Dans la mesure où elle tue l'âme de l'homme civilisé, l'absence du bien-être abrutit. Par ailleurs, l'exemple d'Ithona est inséré pour nous montrer que cet abêtissement ne touche pas l'homme à l'état de nature. L'Indienne que le noble Alphonse épousera est aussi pauvre qu'un Bossendos, plus éloignée qu'une Mathon des bienfaits du confort et du commerce des gens cultivés. Paradoxalement, c'est justement cet éloignement de la civilisation qui lui a permis de conserver ses mours ingénues. Après avoir été initiée à la vie en société par la famille d'Alphonse et les couvents, la belle lthona pourra prendre sa place parmi les prédestinés qui jouissent des grâces du sentiment.

Comme on le voit, la leçon moralisante de Joseph Doutre n'est pas sans obscurités ni contradictions. De plus, elle n'est nullement originale si ce n'est dans son agencement. On retrouvera tous les éléments de la leçon du sentiment du jeune romancier dans d'innombrables moralistes et romanciers philosophes du siècle précédent. A dix-neuf ans, Doutre avait-il lu l'Essai sur le règne de Claude et de Néron de Diderot, De la philosophie de la nature de Delisle de Sales, l'Histoire philosophique et politique des établissements et du commerce des Européens dans les deux Indes de l'abbé de Raynald ou une de ces nombreuses bibles maçonniques sur le plaisir et la vertu? Nous n'en savons rien. Plus probablement, il a dû dévorer plusieurs de ces romans du dix-huitième siècle qui vulgarisaient les apologies du temps sur la vertu facile et l'autorité du sentiment: Les Réflexions d'un jeune homme du chevalier d'Artaize, Le Comte de Valmont de l'abbé Gérard. Les Délices du sentiment 
du chevalier de Mouhy,... Malgré son manque d'originalité tant au niveau didactique qu'au niveau événementiel, l'ouvrage est une œuvre extraordinaire qui nous montre qu'à dix-neuf ans, Joseph Doutre était déjà le libre penseur audacieux qui allait devenir l'ennemi implacable du clergé québécois et de sa morale répressive.

Cependant, à peine émancipé de la tutelle des Sulpiciens (il venait de terminer ses études au collège de Montréal), Doutre n'était pas encore le polémiste affranchi qui allait défendre les libertés de l'Institut Canadien et I'héritage spirituel de Guibord. Dans Les Fiancés de 1812 le romancier affiche, sinon une morale orthodoxe, du moins des justifications exemplaires. Le theme principal, celui de l'amour révolté, nous offre l'exemple d'une charité évangélique qui finit par avoir raison d'une haine démoniaque. Gonzalve essuie les injures de son persécuteur en faisant preuve d'une abnégation héroüque. Finalement, un bon évêque donne aux amants une dispensation qui soustrait Louise à l'autorité despotique de son père. Les illustrations complémentaires sont aussi justificatives. Les mémoires de Gustave nous plongent dans le vice et le crime, mais l'auteur s'évertue à nous démontrer la faiblesse de caractère de son personnage. Ayant sous les yeux l'exemple des amours fidèles de Louise et de Gonzalve, Brandsome, d'abord un cynique débaucheur, se convertit à la moralité des cœurs sensibles. Pour pouvoir épouser Alphonse, Ithona, la jeune Indienne aux mœurs ardentes, endosse la pudeur des couvents. Malgré des justifications qui sont souvent spécieuses, il est clair que Doutre s'efforce de concilier son système moral avec un christianisme aimable. Faut-il y voir une naïveté désarmante ou sommes-nous déjà en présence d'un disciple des libertins qui sait manier l'art de la dissimulation? II nous semble que la morale souriante et les autres précautions du jeune romancier ne peuvent effacer les traits franchement irreligieux et lestes des Fiancés comme le penchant homosexuel de Gustave ou les propos grivois des militaires. L'auteur devait être conscient de la teneur scandalisante de passages comme celui-ci: «... Elle tomba violemment et ses pieds retenus sur le haut de son siège découvrirent tout ce qu'il $y$ a de séduisant chez une femme. Ses compagnes se hâtèrent de la secourir et surtout de voiler le mystère des époux" (p. 450). Même si le roman ne fut pas condamné lors de sa parution 6 , il n'y a nul doute que de tels propos ne furent pas insérés dans le but de se concilier les critiques des Mélanges Religieux ou de l'CEuvre des bons livres des messieurs de Saint-Sulpice.

En dépit de ses faiblesses d'invention et du manque d'originalité de sa morale euphorique, le roman de Doutre est une œuvre importante qui nous apporte un témoignage irremplaçable non seulement sur les premières tendances rationalistes du porte-parole des Rouges mais aussi sur le caractère libertaire et dévergondé du roman québécois avant l'hégémonie ultramontaine des années 1860. Avec le texte original de L'Influence d'un livre d'Aubert de Gaspé fils, la version surprenante de 1849-1851 d'Une de perdue, deux de trouvées de Boucher de Boucherville et Un Épisode de la vie d'un faux-dévot de L'Écuyer, Les Fiancés de 1812 nous apporte la contrepartie, étouffée par 
la tradition casgrainienne, longtemps insoupçonnée, des œuvres édifiantes et des propos moralisants des Lacombe, des Chauveau, des Olivier et des Cherrier.

1. Voir Les Lettres canadiennes d'autrefois, Ottawa, Editions de l'Université d'Ottawa, 1944, tome IV, pp. 60-70 surtout.

2. Voir «Les Origines du roman canadien-français» dans Le Roman canadien-français, Montréal, Fides, Archives des lettres canadiennes, III, 1964, pp. 37-67.

3. Notre pagination provient de l'édition originale, Les Fiancés de 1812, Montréal, Perrault, 1844.

4. Nous empruntons l'expression "autorité du sentiment" et sa connotation d'ascendance à Jean Starobinski. Voir "Critiquè et principe d'autorité » dans Mouvements premiers. Études critiques offertes à Georges Poulet, Paris, José Corti, 1972, pp. 87-106.

5. Robert Mauzi, L'dée du bonheur dans la littérature et la pensée française au XVIIIe siècle, Paris, Librairie Armand Colin, 1960, p. 595.

6. La première condamnation des Fiancés date de 1848 , quatre ans après sa parution. Voir L. L., "Chronique littéraire" dans Les Mélanges Religieux, XII, 17 (8 nov. 1848), 67 et, Le chroniqueur, "Nos lecteurs doivent se rappeler..." dans Les Mélanges Religieux, XII, 19 (17 nov. 1848), 74. Cette critique acerbe semble avoir été déclenchée moins par un renouveau d'intérêt pour le roman que par la guerre entre L'Avenir de Doutre et La Minerve de Louis-Octave Létourneux [L.L.?]. 